\title{
General Psychiatry Increased plasma leptin as a novel predictor for psychopathological depressive symptoms in chronic schizophrenia
}

Jinjie Xu, ${ }^{1,2,3}$ Yumei Jiao, ${ }^{1}$ Mengjuan Xing, ${ }^{1}$ Yezhe Lin, ${ }^{1}$ Yousong Su, ${ }^{1}$
Wenhua Ding, ${ }^{1}$ Cuizhen Zhu, ${ }^{1}$ Yanmin Peng,,${ }^{1}$ Dake Qi,,${ }^{1,4}$ Donghong Cui ${ }^{1,2,5}$
To cite: $X u$ J, Jiao Y, Xing M, et al. Increased plasma leptin as a novel predictor for psychopathological depressive symptoms in chronic schizophrenia. General Psychiatry 2018;31:e100018. doi:10.1136/

gpsych-2018-100018

$J X$ and $Y J$ contributed equally.

Received 24 September 2018 Revised 16 November 2018 Accepted 18 November 2018

Check for updates

(C) Author(s) (or their employer(s)) 2018 . Re-use permitted under CC BY-NC. No commercial re-use. See rights and permissions. Published by BMJ.

${ }^{1}$ Shanghai Mental Health Center, Shanghai Jiao Tong University, School of Medicine, Shanghai, China

${ }^{2}$ Shanghai Key Laboratory of Psychotic Disorders, Shanghai, China

${ }^{3}$ Beijing Anding Hospital, Capital Medical University, Beijing,

China

${ }^{4}$ Division of Biomedical

Sciences, Faculty of Medicine,

Memorial University of

Newfoundland, St. John's,

Newfoundland and Labrador,

Canada

${ }^{5}$ Brain Science and Technology Research Center, Shanghai Jiao Tong University, Shanghai, China

Correspondence to

Dr Donghong Cui;

manyucc@126.com

\section{ABSTRACT}

Background Depressive symptoms are often seen in schizophrenia. The overlap in presentation makes it difficult to distinguish depressive symptoms from the negative symptoms of schizophrenia. The adipokine leptin was found to be altered in both depression and schizophrenia. There are few studies focusing on the prediction of leptin in diagnosis and evaluation of depressive symptoms in schizophrenia.

ObjectiveAims To assess the plasma leptin level in patients with schizophrenia and its relationships with depressive symptoms.

Methods Cross-sectional studies were applied to (1) compare the levels of plasma leptin between schizophrenia $(n=74)$ and healthy controls $(n=50)$; and (2) investigate the relationship between plasma leptin levels and depressive subscores.

Results (1) Plasma leptin levels were significantly higher in patients with schizophrenia than in healthy controls. (2) Correlation analysis revealed a significant negative association between leptin levels and the depressed factor scores on the Positive and Negative Syndrome Scale (PANSS). (3) Stepwise multiple regression analyses identified leptin as an influencing factor for depressed factor score on PANSS.

Conclusion Leptin may serve as a predictor for the depressive symptoms of chronic schizophrenia.

\section{INTRODUCTION}

Schizophrenia is a chronic and severe mental disorder with a lifetime prevalence of around $1 \%$ globally. ${ }^{1}$ It has a profound impact on individuals, families and society. Over half of the patients with schizophrenia suffer from intermittent psychosis and approximately one-fifth of them have chronic symptoms, and mental and physical disability. ${ }^{2}$ Schizophrenia is characterised by diverse psychopathology with symptoms that are commonly divided into five dimensions: positive symptoms, negative symptoms, cognitive symptoms, aggressive symptoms and affective symptoms. ${ }^{3}$ Depressive symptoms (seen as one end on the spectrum of affective symptoms) frequently accompany schizophrenia, may be part of the prodromal symptomology, following an acute episode, or occur between psychotic episodes. ${ }^{4}$ Although not as dramatic as positive and aggressive symptoms, it should be noted that the depressive symptoms of schizophrenia have been reported to be associated with various less favourable outcomes in schizophrenia. ${ }^{5-7}$ These include poorer responses to medication, greater impairments in social and vocational functioning, more personal stigma, higher risk of discontinuation of medication and rehospitalisation, longer durations of hospitalisation, greater family and societal burden, more negative life events and even increased rate of suicide attempts and completed suicides. ${ }^{8-10}$ Unfortunately, less attention is paid to depressive symptoms than other symptom domains of schizophrenia. To make matters worse, it is quite difficult to distinguish a depressive syndrome from other diagnoses with overlapping features, in particular the negative symptoms commonly seen in schizophrenia. The negative syndrome includes a number of clinical presentations which are similar to components of the depression syndrome, such as anhedonia, anergia, alogia, blunted affect, social withdrawal and loss of motivation, which may confuse doctors. ${ }^{11}{ }^{12}$ Accordingly, patients may not receive adjunctive antidepressant treatment in good time. Therefore, a biomarker for depressive syndromes is of great interest.

Leptin, encoded by the obese (ob) gene, is a $16 \mathrm{kD}$ protein primarily synthesised and secreted by adipocytes. It exerts multiple biological functions by binding to its receptors. Initially, leptin was found to inhibit food intake and promote energy consumption and therefore it was known as an antiobesity hormone. ${ }^{13}$ However, the function of leptin 
85 patients with schizophrenia at the Shanghai Mental Health Center.

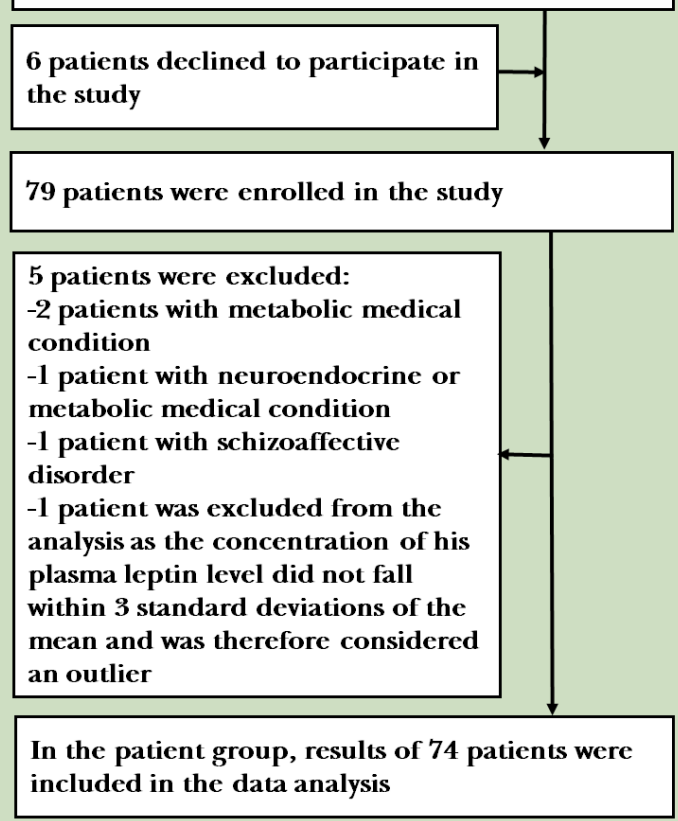

52 volunteers were recruited via advertisements and social contact during the same period

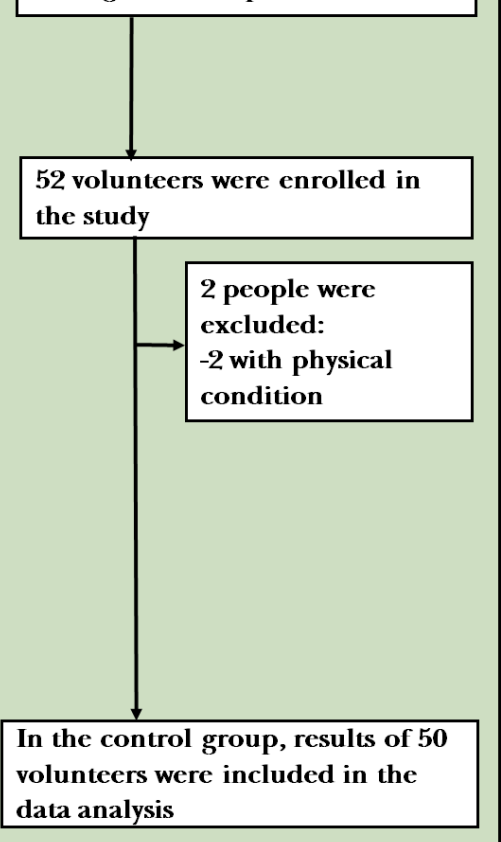

Figure 1 The flow chart of the study.

has recently been broadened into involvement of mood disorders, in particular depression. ${ }^{14-17}$ Ample literature has indicated leptin as a novel modulator of depressive mood. Animal studies showed that leptin receptors were highly expressed in mood-related brain regions including the amygdala, cortex and hippocampus. ${ }^{18} \mathrm{Clin}-$ ical studies found decreased leptin levels in patients with major depression and observed increases in leptin levels accompanied with improvements in depressive symptoms following antidepressant treatment. ${ }^{19-22}$ Therefore, plasma leptin may serve as a peripheral biomarker for the differential diagnosis and evaluation of depressive syndromes in schizophrenia.

In light of the findings mentioned above, we hypothesised that the plasma leptin levels may be associated with depressive symptoms in chronic schizophrenia. Therefore, our present study aims to investigate (1) whether plasma leptin levels were altered in patients with schizophrenia; (2) whether there was a relationship between plasma leptin levels and psychopathological parameters, particularly the depressive subscore.

\section{METHODS}

\section{Subjects}

Eighty-five inpatients were recruited from January 2017 to June 2017, and 52 healthy controls matched for gender, age and body mass index (BMI) were recruited by advertisement (figure 1).

The inclusion criteria for patients were the following: (1) meeting diagnostic criteria for schizophrenia according to the Diagnostic and Statistical Manual of Mental Disorders, Fourth Edition based on the Mini-International Neuropsychiatric Interview; (2) aged 18-80, ethnically Han Chinese; (3) had a duration of schizophrenia $\geq 4$ years; (4) experienced multiple psychotic episodes $\geqq 2$ times; and (5) on stable antipsychotic treatment with either chlorpromazine or olanzapine $\geqq 12$ months (without dose change for at least 30 days). None of the participants in the control group had a personal or family history of psychotic disorders as assessed by two experienced psychiatrists.

The exclusion criteria were the following: (1) were diagnosed with schizoaffective disorder, mental retardation, substance abuse, cognitive disorders or other mental disorder; (2) had a severe neuroendocrine or metabolic medical condition, or immunologic alterations other than those related to antipsychotics; and (3) were pregnant or nursing.

After strict screening, 75 patients were included in our study while 10 were excluded for the following reasons: six patients declined to participate in, three patients had metabolic or neuroendocrine disease and one patient was diagnosed with schizoaffective disorder. Fifty healthy controls were included in the study while two were excluded because of physical condition (as shown in figure 1).

All participants provided written informed consent. Demographic data, physical examination and blood samples were collected from all subjects. 


\section{Clinical evaluation}

The patients' psychopathology was evaluated by two psychiatrists with clinical experience for more than or equal to 5 years, using the Positive and Negative Syndrome Scale (PANSS) ${ }^{23}$ and the Brief Psychiatric Rating Scale (BPRS).$^{2324}$ Training consistency of PANSS and BPRS was conducted between the two psychiatrists before the evaluation. Thereafter, an intraclass correlation coefficient $\geqq 0.8$ was maintained at repeated evaluation in this study.

Although the items of PANSS were originally grouped into three subscales, ${ }^{25}$ factor analyses indicated that a fivefactor model may better characterise PANSS data: positive factor (items P1, P3, P5, G9), negative factor (items N1, N2, N3, N4, N6, G7), cognitive factor (items P2, N5, G11), excited factor (items P4, P7, G8, G14) and depressed factor (items G2, G3, G6). ${ }^{2627}$

\section{Measurement of plasma leptin, glucose and lipid profiles}

All blood samples were collected from subjects between 07:00 and 09:00 following an overnight fast. Plasma was then separated, aliquoted and stored at $-80^{\circ} \mathrm{C}$. Plasma leptin levels were measured in triplicate by using commercial ELISA kit (R\&D Systems, USA). All samples were assayed by the same technician who was blind to the clinical status of the samples. The interassay and intra-assay coefficients of variability for leptin were $3.4 \%$ and $4.5 \%$, respectively.

\section{Statistical analysis}

Statistical analysis was performed by using the Statistical Package for Social Sciences (SPSS, V.23.0; IBM).
The Kolmogorov-Smirnov test was used to examine the normal distribution of data. Because of the non-normal distribution, the concentration of leptin was converted into $\lg$ (leptin) before further statistical analysis. Independent samples t-test for continuous variables and $\chi^{2}$ test for categorical variables were respectively used to compare demographic data and clinical variables between groups. Analysis of covariance (ANCOVA) was further used to control for confounding effects of gender, age and BMI on leptin levels. Spearman's $r$ test was used to examine the correlation between leptin and psychopathological variables when controlled for gender, age and BMI. Multiple stepwise regression analysis was then used to identify significant predictive variables associated with psychopathological variables when controlled for gender, age and BMI. In addition, Bonferroni correction was applied to adjust for multiple testing. All $\mathrm{p}$ values were two tailed with the significance level set at 0.05 .

\section{RESULTS}

Demographic data of patients with schizophrenia and healthy controls

A total of 75 patients with schizophrenia and 50 healthy subjects were included in this study. One patient was excluded from the analysis as the concentration of his plasma leptin level did not fall within 3 SDs of the mean and was therefore considered an outlier. Hence all analyses were performed on 74 patients and 50 controls. As

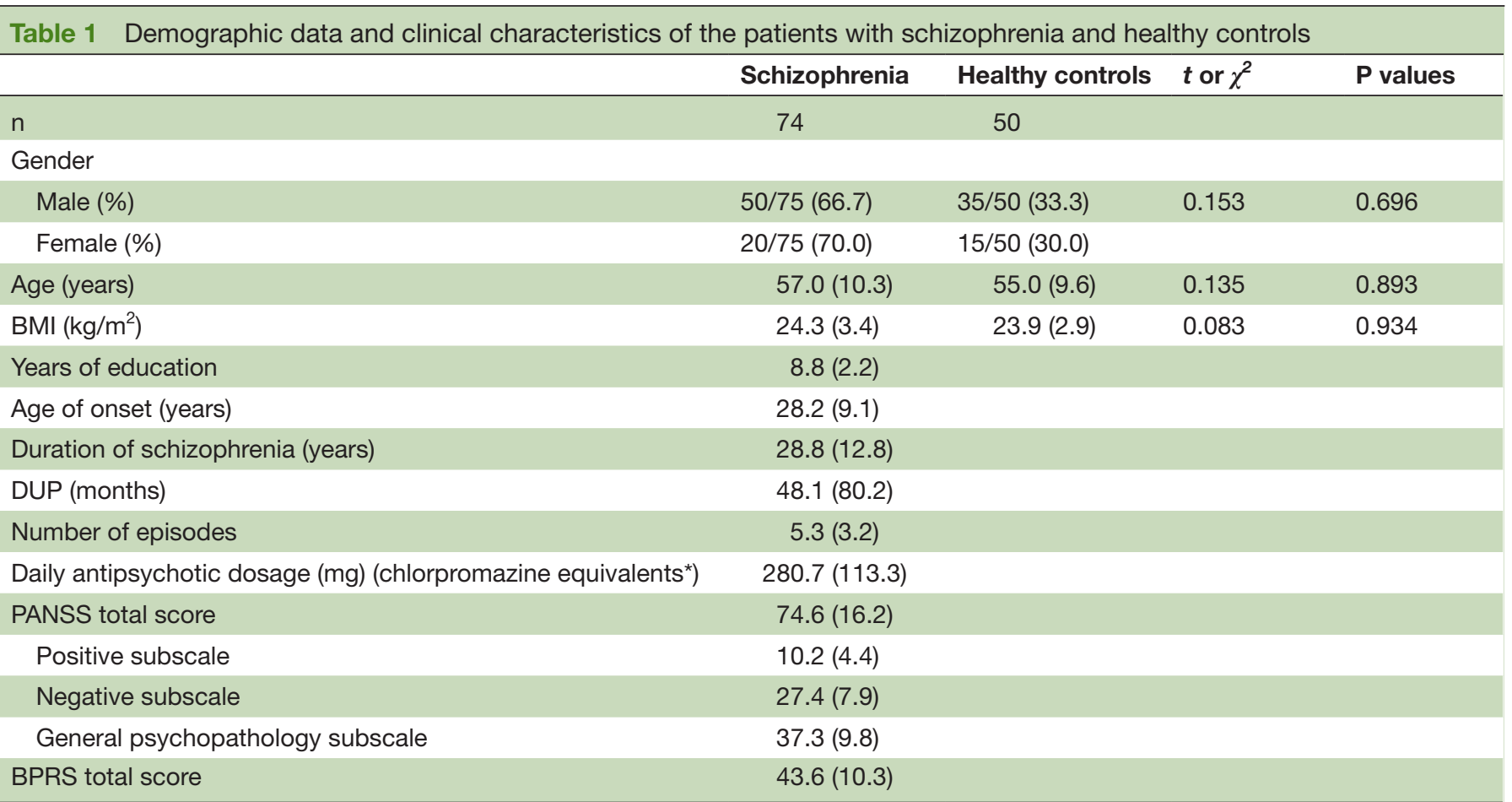

*The chlorpromazine equivalents were calculated as described by Gray et al. ${ }^{42}$

BMI, body mass index; BPRS, Brief Psychiatric Rating Scale; DUP, duration of untreated psychosis; PANSS, Positive and Negative Syndrome Scale. 


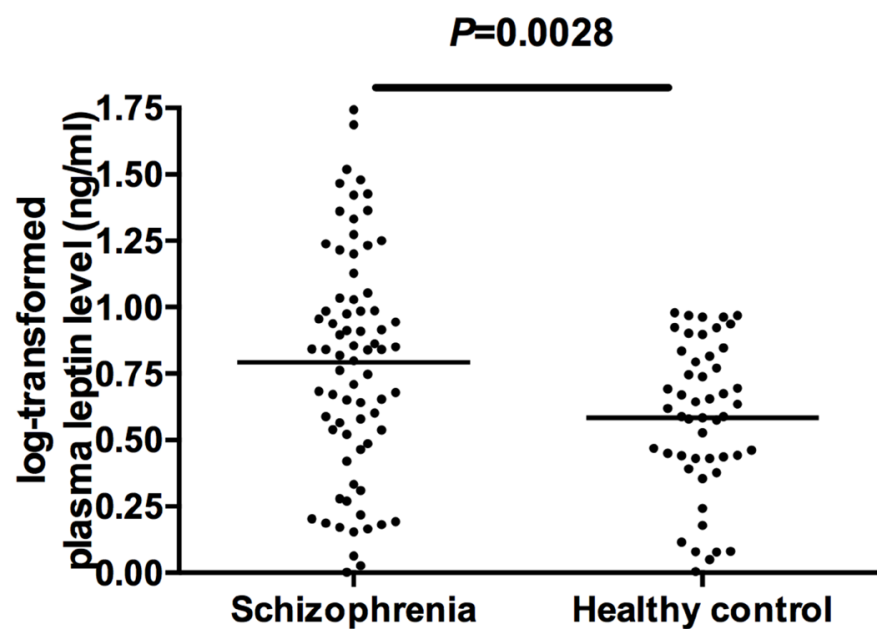

Figure 2 Logarithmic transformed plasma leptin levels (lg leptin) in patients with schizophrenia $(n=74)$ and healthy controls $(n=50)$. The sample means are indicated by the black bars. Plasma lg leptin levels were significantly higher in patients with schizophrenia than healthy controls.

shown in table 1, no significant differences in gender, age and BMI were found between the patients and controls.

\section{Plasma leptin levels in patients with schizophrenia and} healthy controls

Logarithmic transformed plasma leptin levels (lg leptin) were significantly higher in patients with schizophrenia than in healthy controls (figure 2). When gender, age and BMI were added as potential confounding covariates to ANCOVA, the differences between groups remained significant $(F=25.79, \mathrm{df}=1, p<0.001)$.

\section{Association between leptin levels and psychopathology in schizophrenia}

When controlled for gender, age and BMI, partial correlation analysis indicated a significantly negative association between plasma leptin levels and the PANSS depression factor scores $(r=-0.255$, Bonferroni corrected $p=0.028$, as shown in table 2).

Table 2 Partial correlations between leptin levels and clinical variables in patients with schizophrenia, controlled for gender, age and BMI (Spearman's r) ( $n=74)$

\begin{tabular}{ccl}
\hline & Correlation coefficient & P values \\
\hline PANSS total score & -0.039 & 0.745 \\
Positive factor & 0.141 & 0.230 \\
Negative factor & -0.970 & 0.412 \\
Cognitive factor & -0.045 & 0.707 \\
Excited factor & 0.139 & 0.236 \\
\hline Depressed factor & -0.255 & $0.028^{\star}$ \\
BPRS total score & -0.074 & 0.535 \\
\hline
\end{tabular}

*Indicated the Bonferroni corrected $p$ value, $p<0.05$.

BMI, body mass index; BPRS, Brief Psychiatric Rating Scale;

PANSS, Positive and Negative Syndrome Scale.
Table 3 Partial correlations between leptin levels and clinical variables in schizophrenia with depressive symptoms, controlled for gender, age and BMI (Spearman's r) $(n=47)$

\begin{tabular}{ccl}
\hline & Correlation coefficient & P values \\
\hline PANSS total score & 0.008 & 0.958 \\
Positive factor & 0.23 & 0.121 \\
Negative factor & -0.069 & 0.644 \\
Cognitive factor & 0.069 & 0.644 \\
Excited factor & 0.373 & 0.352 \\
Depressed factor & -0.303 & $0.038^{*}$ \\
BPRS total score & -0.04 & 0.789 \\
\hline
\end{tabular}

*Indicated the Bonferroni corrected $p$ value, $p<0.05$. BMI, body mass index; BPRS, Brief Psychiatric Rating Scale; PANSS, Positive and Negative Syndrome Scale.

Furthermore, a multiple stepwise regression was run to predict the PANSS depression factor with leptin levels and demographics including years of education, age of onset, duration of schizophrenia, duration of untreated psychosis, number of episodes and daily antipsychotic dosage. When adjusted for gender, age and BMI, leptin levels were identified as a significant predictor for the PANSS depression factor $(\beta=-0.33, \quad t=-2.32, \quad p=0.024)$ $(\beta=-0.26, t=-1.79, p=0.032)$.

According to the criteria of PANSS depression factor score $>3$, forty-seven of the 74 patients with schizophrenia had depressive symptoms. In the depressive symptom group, correlation analysis $(r=-0.303$, Bonferroni corrected $p=0.038$, as shown in table 3 ) and regression analysis $(\beta=-0.26, t=-1.79, p=0.032)$ further confirmed that plasma leptin levels were significantly negatively associated with the PANSS depressed factor.

\section{DISCUSSION}

\section{Main findings}

This study had two main findings. (1) Plasma leptin levels were significantly increased in patients with schizophrenia compared with the healthy controls despite controlling for gender, age and BMI. (2) Plasma leptin levels were significantly negatively associated with the PANSS depressed factor but not with other factor components.

In this study, we observed significantly higher plasma leptin levels in patients with chronic schizophrenia, which was in agreement with previous studies. ${ }^{162829}$ Early research focused primarily on the relationship between leptin levels and weight changes associated with antipsychotic treatment. Many studies reported that the elevated leptin levels in patients with schizophrenia were largely dependent on weight gain and BMI. However, few of these studies considered that a difference in BMI existed between groups when they compared leptin levels. On the contrary, gender, age and BMI were taken into account, and the difference of leptin levels between the patients and controls remained significant. Likewise, Kim 
and colleagues recently found that plasma leptin levels tended to be higher in patients with schizophrenia than in age-matched and BMI-matched healthy controls. ${ }^{30} \mathrm{~A}$ recent meta-analysis also found that schizophrenia was associated with elevated blood leptin levels compared with controls, but the difference in leptin levels between groups was not moderated by BMI. Taken together, these studies suggest that leptin levels may be associated with multiple factors, for example, the psychopathology of schizophrenia, different phases of illness and lifestyles.

In our present study, it was further found that there was a significant negative correlation between plasma leptin levels and the PANSS depressed factor subscore in patients with schizophrenia. Previous studies observed decreased plasma leptin levels in patients with or without comorbid major depression compared with controls. ${ }^{31} 32$ Furthermore, studies found that the negative relationship between leptin levels and the severity of depressive symptoms was independent of body weight or BMI. ${ }^{28} 3334$ In consistency with these findings, our study also found a link between leptin levels and depressive symptoms in patients with schizophrenia after controlling for BMI.

\section{Limitations}

Several limitations of this study should be noted. First, due to the nature of cross-sectional studies, the patients' baseline information was unknown (eg, plasma leptin levels, psychopathological symptoms and previous medication use) and therefore the results obtained in this study may be confounded. Second, as the sample size is small and leptin level is fluctuated, our findings should be accordingly considered as preliminary exploration. Last, it is worth mentioning that the depressed factor used in the present study is derived from the five-factor model of PANSS scale, which is used for symptomatology. A specific depression scale such as Hamilton Depression Rating Scale may have been a better choice. Therefore, in order to get a conclusive and not just suggestive result, the relationship between leptin and depressive symptomology in schizophrenia deserves further investigation using a specific depression scale in a large-scale longitudinal study, or even a randomised, double-blind and parallel controlled clinical trial.

\section{Implications}

Different theories have been proposed in the literature to explain the negative relationship between plasma leptin levels and the depressive symptoms.

First, leptin may be involved in regulation of serotonin (5-HT) and dopamine (DA), which are the key neurotransmitters in the classic aetiological hypothesis of depression. Previous studies have found that intraperitoneal administration of leptin dose dependently significantly increased serum 5-HT levels, ${ }^{35}$ while leptin-deficient $o b /$ $o b$ mice exhibited impaired DA functions and decreased DA release. ${ }^{36}$ Second, leptin may have direct and indirect neurotrophic effects, which are disrupted in depression. Previous studies showed that leptin administration elevated the expression of brain-derived neurotrophic factor, one of the most important neurotrophic factors. ${ }^{37}$ Leptin-deficient $o b / o b$ mice showed decreased weight overall for the whole brain and decreased volume of specific brain regions, while these neurodegenerative changes were partially reversed by leptin treatment. ${ }^{35} 37-40$ Third, leptin may be a potent inhibitor of the hypothalamic-pituitary-adrenal axis, which is overactive in depression. $^{41}$

To our knowledge, this is the first study to explore the relationship between leptin levels and depressive symptoms in schizophrenia, while previous studies focused mainly on major depressive disorder. If our results could be confirmed in further large-scale longitudinal studies, plasma leptin level would probably serve as a promising predictor for depressive symptoms in schizophrenia.

Acknowledgements The authors gratefully acknowledge all subjects for their participation in the current study.

Authors' contributions DC and DQ were responsible for study design and revising. JX, MX, YL, YS and WD were responsible for clinical data collection and lab experiments. JX, YJ and DQ managed the literature search and the statistical analyses. JX drafted the manuscript. DC, DQ, YP and CZ participated in revising the manuscript. All authors read and approved the final manuscript.

Funding This study was supported by grants from the National Key R\&D Program of China (2017YFC0909200), the National Science Foundation of China (NSFC; $81171266,81271481,81671336$ and 81500976), the China and National Key Research and Development Program (2017YFC0909200), the Shanghai Key Laboratory of Psychotic Disorders (13dz2260500), the Shanghai Municipal Planning Commission of Science and Research Fund (20154Y0194) and the Canadian Institutes of Health Research (project grant PJT-156116).

Competing interests None declared.

Informed consent Obtained.

Ethics approval Institutional Review Board of Shanghai Mental Health Center. Provenance and peer review Not commissioned; externally peer reviewed.

Data sharing statement

No additional data are available.

Open access This is an Open Access article distributed in accordance with the Creative Commons Attribution Non Commercial (CC BY-NC 4.0) license, which permits others to distribute, remix, adapt, build upon this work non-commercially, and license their derivative works on different terms, provided the original work is properly cited and the use is non-commercial. See: http://creativecommons.org/ licenses/by-nc/4.0

\section{REFERENCES}

1. Association AP. Diagnostic and statistical manual of mental disorders (DSM-5). American Psychiatric Pub, 2013.

2. Barbato A. Psychiatry in transition: outcomes of mental health policy shift in Italy. Aust N Z J Psychiatry 1998;32:673-9.

3. Stahl SM. Stahl's essential psychopharmacology: neuroscientific basis and practical applications. Cambridge university press, 2013.

4. Planansky K, Johnston R. Depressive syndrome in schizophrenia. Acta Psychiatr Scand 1978;57:207-18.

5. Conus P, Abdel-Baki A, Harrigan S, et al. Pre-morbid and outcome correlates of first episode mania with psychosis: is a distinction between schizoaffective and bipolar I disorder valid in the early phase of psychotic disorders? J Affect Disord 2010;126:88-95.

6. Conley RR, Ascher-Svanum H, Zhu B, et al. The burden of depressive symptoms in the long-term treatment of patients with schizophrenia. Schizophr Res 2007;90:186-97.

7. Buckley PF, Miller BJ, Lehrer DS, et al. Psychiatric comorbidities and schizophrenia. Schizophr Bull 2009;35:383-402.

8. Siris SG. Suicide and schizophrenia. J Psychopharmacol 2001;15:127-35. 
9. Palmer BA, Pankratz VS, Bostwick JM. The lifetime risk of suicide in schizophrenia: a reexamination. Arch Gen Psychiatry 2005;62:247-53.

10. Hawton K, Sutton L, Haw C, et al. Schizophrenia and suicide: systematic review of risk factors. Br J Psychiatry 2005;187:9-20.

11. Carpenter WT, Heinrichs DW, Alphs LD. Treatment of negative symptoms. Schizophr Bull 1985;11:440-52.

12. Andreasen NC, Olsen S. Negative v positive schizophrenia. Definition and validation. Arch Gen Psychiatry 1982;39:789-94.

13. Elmquist JK, Bjørbaek C, Ahima RS, et al. Distributions of leptin receptor mRNA isoforms in the rat brain. $J$ Comp Neurol 1998;395:535-47.

14. Pan W, Kastin AJ. Leptin: a biomarker for sleep disorders? Sleep Med Rev 2014;18:283-90.

15. Tezapsidis N, Johnston JM, Smith MA, et al. Leptin: a novel therapeutic strategy for Alzheimer's disease. J Alzheimers Dis 2009;16:731-40.

16. Sentissi O, Epelbaum J, Olié JP, et al. Leptin and ghrelin levels in patients with schizophrenia during different antipsychotics treatment: a review. Schizophr Bull 2008;34:1189-99.

17. Casanueva FF, Dieguez C, Popovic V, et al. Serum immunoreactive leptin concentrations in patients with anorexia nervosa before and after partial weight recovery. Biochem Mol Med 1997;60:116-20.

18. Lu XY. The leptin hypothesis of depression: a potential link between mood disorders and obesity? Curr Opin Pharmacol 2007;7:648-52.

19. Schilling C, Gilles M, Blum WF, et al. Leptin plasma concentrations increase during antidepressant treatment with amitriptyline and mirtazapine, but not paroxetine and venlafaxine: leptin resistance mediated by antihistaminergic activity? J Clin Psychopharmacol 2013;33:99-103.

20. Esel E, Ozsoy S, Tutus A, et al. Effects of antidepressant treatment and of gender on serum leptin levels in patients with major depression. Prog Neuropsychopharmacol Biol Psychiatry 2005;29:565-70.

21. Pasco JA, Jacka FN, Williams LJ, et al. Leptin in depressed women: cross-sectional and longitudinal data from an epidemiologic study. $J$ Affect Disord 2008;107(1-3):221-5.

22. Kraus T, Haack M, Schuld A, et al. Low leptin levels but Norma body mass indices in patients with depression or schizophrenia. Neuroendocrinology 2001;73:243-7.

23. Kay SR, Fiszbein A, Opler LA, eds. Positive and negative syndrome scale (PANSS). MHS, 2012

24. Faustman WO, Overall JE. Brief psychiatric rating scale, 1999.

25. Kay SR, Fiszbein A, Opler LA. The positive and negative syndrome scale (PANSS) for schizophrenia. Schizophr Bull 1987;13:261-76.

26. Citrome L, Meng X, Hochfeld M. Efficacy of iloperidone in schizophrenia: a PANSS five-factor analysis. Schizophr Res 2011;131:75-81.
27. Wallwork RS, Fortgang R, Hashimoto R, et al. Searching for a consensus five-factor model of the Positive and Negative Syndrome Scale for schizophrenia. Schizophr Res 2012;137:246-50.

28. Jow GM, Yang TT, Chen CL. Leptin and cholesterol levels are low in major depressive disorder, but high in schizophrenia. J Affect Disord 2006;90:21-7.

29. Melkersson KI, Hulting AL, Brismar KE. Elevated levels of insulin, leptin, and blood lipids in olanzapine-treated patients with schizophrenia or related psychoses. J Clin Psychiatry 2000;61:742-9.

30. Kim JH, Kim JH, Park PW, et al. Body and liver fat content and adipokines in schizophrenia: a magnetic resonance imaging and spectroscopy study. Psychopharmacology 2017;234:1923-32.

31. Atmaca M, Kuloglu M, Tezcan E, et al. Serum leptin and cholesterol levels in patients with bipolar disorder. Neuropsychobiology 2002;46:176-9.

32. Emül HM, Serteser M, Kurt E, et al. Ghrelin and leptin levels in patients with obsessive-compulsive disorder. Prog Neuropsychopharmacol Biol Psychiatry 2007;31:1270-4.

33. Kraus T, Haack M, Schuld A. Low leptin levels but normal body mass indices in patients with depression or schizophrenia. Neuroendocrinology 2001;7354641:243-7.

34. Lawson EA, Miller KK, Blum Jl, et al. Leptin levels are associated with decreased depressive symptoms in women across the weight spectrum, independent of body fat. Clin Endocrinol 2012;76:520-5.

35. Haleem DJ, Haque Z, Inam QU, et al. Behavioral, hormonal and central serotonin modulating effects of injected leptin. Peptides 2015;74:1-8.

36. Fulton S, Pissios P, Manchon RP, et al. Leptin regulation of the mesoaccumbens dopamine pathway. Neuron 2006;51:811-22.

37. Komori T, Morikawa Y, Nanjo K, et al. Induction of brain-derived neurotrophic factor by leptin in the ventromedial hypothalamus. Neuroscience 2006;139:1107-15.

38. Farr SA, Banks WA, Morley JE. Effects of leptin on memory processing. Peptides 2006;27:1420-5.

39. Drel VR, Mashtalir N, Inytska O, et al. The leptin-deficient (ob/ob) mouse: a new animal model of peripheral neuropathy of type 2 diabetes and obesity. Diabetes 2006;55:3335-43.

40. Schwartz MW, Baskin DG, Bukowski TR, et al. Specificity of leptin action on elevated blood glucose levels and hypothalamic neuropeptide $\mathrm{Y}$ gene expression in ob/ob mice. Diabetes 1996;45:531-5.

41. Flak JN. A role for leptin-regulated neurocircuitry in subordination stress. Physiol Behav 2017;178:144-50.

42. Gray $\mathrm{AH}$, Wright J, Bruce L. Clinical pharmacy pocket companion. Pharmaceutical Press, 2015.

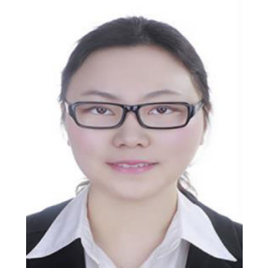

Jinjie Xu graduated with a bachelor's degree from Nanjing Medical University in 2013 and a master's degree from Shanghai Mental Health Center, Shanghai Jiao Tong University, School of Medicine in 2017. She began her residency at Beijing Anding Hospital in 2017 and is now a full-time resident doctor. Her main interests are clinical studies of mental disorders. 